\title{
Pig Model of Pulmonary Embolism: Where Is the Hemodynamic Break Point?
}

\author{
J. KUDLIČKA ${ }^{1,2}$, M. MLČEK ${ }^{1}$, P. HÁLA ${ }^{1,3}$, S. LACKO $^{1}$, D. JANÁK ${ }^{1,4}$, M. HRACHOVINA ${ }^{1}$, \\ J. MALÍK ${ }^{2}$, J. BĚLOHLÁVEK ${ }^{5}$, P. NEUŽIL ${ }^{3}$, O. KITTNAR ${ }^{1}$
}

${ }^{1}$ Institute of Physiology, First Faculty of Medicine, Charles University in Prague, Czech Republic,

${ }^{2}$ Third Department of Medicine, First Faculty of Medicine, Charles University in Prague and General University Hospital, Prague, Czech Republic, ${ }^{3}$ Department of Cardiology, Na Homolce Hospital, Prague, Czech Republic, ${ }^{4}$ Second Department of Surgery, First Faculty of Medicine, Charles University in Prague and General University Hospital, Prague, Czech Republic, ${ }^{5}$ Second Department of Medicine, First Faculty of Medicine, Charles University in Prague and General University Hospital, Prague, Czech Republic

Received March 18, 2013

Accepted September 4, 2013

\section{Summary}

Early recognition of collapsing hemodynamics in pulmonary embolism is necessary to avoid cardiac arrest using aggressive medical therapy or mechanical cardiac support. The aim of the study was to identify the maximal acute hemodynamic compensatory steady state. Overall, 40 dynamic obstructions of pulmonary artery were performed and hemodynamic data were collected. Occlusion of only left or right pulmonary artery did not lead to the hemodynamic collapse. When gradually obstructing the bifurcation, the right ventricle end-diastolic area expanded proportionally to pulmonary artery mean pressure from 11.6 $(10.1,14.1)$ to $17.8(16.1,18.8) \mathrm{cm}^{2}(\mathrm{p}<0.0001)$ and pulmonary artery mean pressure increased from $22(20,24)$ to $44(41,47)$ $\mathrm{mmHg}(p<0.0001)$ at the point of maximal hemodynamic compensatory steady state. Similarly, mean arterial pressure decreased from $96(87,101)$ to $60(53,78) \mathrm{mmHg}(\mathrm{p}<0.0001)$, central venous pressure increased from $4(4,5)$ to $7(6,8)$ $\mathrm{mmHg}(\mathrm{p}<0.0001)$, heart rate increased from $92(88,97)$ to 147 $(122,165) / \mathrm{min}(\mathrm{p}<0.0001)$, continuous cardiac output dropped from $5.2(4.7,5.8)$ to $4.3(3.7,5.0) \mathrm{l} / \mathrm{min}(\mathrm{p}=0.0023)$, modified shock index increased from $0.99(0.81,1.10)$ to 2.31 (1.99, $2.72), p<0.0001$. In conclusion, instead of continuous cardiac output all of the analyzed parameters can sensitively determine the individual maximal compensatory response to obstructive shock. We assume their monitoring can be used to predict the critical phase of the hemodynamic status in routine practice.

\section{Key words}

Pulmonary embolism • Hemodynamics • Shock • Pig model

\section{Corresponding author}

Jaroslav Kudlicka, 3rd Dept. of Medicine, General University Hospital, U Nemocnice 1, 12808 Prague, Czech Republic. E-mail: jaroslav.kudlicka@vfn.cz

\section{Introduction}

Pulmonary embolism (PE) belongs to common cardiovascular emergencies and may lead to lifethreatening right ventricle dysfunction. Therefore, early diagnosis and immediate treatment is fundamental (Torbicki et al. 2008). Especially massive or high risk PE presented with cardiac arrest or severe systemic hypotension (systolic blood pressure $<100 \mathrm{mmHg}$ ) is associated with very high mortality (Aujesky et al. 2005, Kasper et al. 1997, Wicki et al. 2000). The first-line treatment of patients with high risk PE is thrombolytic therapy, which can rapidly eliminate thromboembolic obstruction of the pulmonary artery (Dalla-Volta et al. 1992, Levine et al. 1990). When the thrombolysis is contraindicated or fails to improve hemodynamics, other lifesaving procedures should be considered: acute surgical embolectomy or catheter based interventions (Leacche et al. 2005, Meneveau et al. 2006, Sukhija et al. 2005). In these patients, intensive hemodynamic and 
respiratory support is necessary to overcome critical systemic hypotension and to prevent the cardiac arrest (Kearon et al. 2008, Torbicki et al. 2008). Besides the aggressive pharmacotherapy (including administration of catecholamines), some case reports (Davies et al. 1995, Hsieh et al. 2001, Weinberg et al. 2011) and first small study (Malekan et al. 2012) suggest the use of peripheral extracorporeal membrane oxygenation (ECMO) as a bridge to the ultimate solution. On the other hand, ECMO also carries the risk of complications such as cannulation related difficulties, severe bleeding, systemic air embolism and infection (Bartlett et al. 2010). Appropriate risk stratification of $\mathrm{PE}$ and timing of therapeutic procedures is therefore crucial for the patient's outcome.

It is known that hemodynamic consequences appear when at least $30-50 \%$ of pulmonary arterial bed is occluded (McIntyre et al. 1971). Sympathetic response together with administered catecholamines and Frank Starling mechanism help the right ventricle (RV) to restore pulmonary flow, which improves left ventricle filling and cardiac output. Systemic vasoconstriction leads to higher central arterial pressures, which are important for adequate coronary perfusion (Molloy et al. 1984). Destabilization of compensatory response may occur after recurrent embolization or by long term oxygen depletion and/or myocardial perfusion reduction.

The aim of this animal study was to describe complex hemodynamic changes in dynamic obstruction of pulmonary artery and to find out the point of maximal acute hemodynamic compensatory response, which could be extremely helpful in urgent therapeutic strategies decision.

\section{Methods}

The protocol was designed to simulate initiation and development of severe acute pulmonary embolism by obstructing pulmonary artery with a balloon catheter. All procedures were performed on cross-bred swine (Landrace $\mathrm{x}$ White) in accredited university experimental laboratory by the skilled team of intensive care specialists. The experimental protocol was approved by the institutional Animal Expert Committee of Charles University. Overall, 5 animals were used (female, age 4-5 months, mean weight $51 \pm 2 \mathrm{~kg}$ ).

\section{Anesthesia and monitoring}

After intramuscular sedation and premedication (azaperone 2-3 $\mathrm{mg} / \mathrm{kg}$, atropine $10 \mu \mathrm{g} / \mathrm{kg}$, ketamine
$20 \mathrm{mg} / \mathrm{kg}$ all i.m.), the marginal ear vein was cannulated and the pigs were preoxygenated with $100 \%$ oxygen via a facial mask. General anesthesia was induced by intravenous bolus of propofol $(2 \mathrm{mg} / \mathrm{kg})$ and orotracheal intubation was performed. Mechanical ventilation was adjusted by Intellivent-ASV closed-loop system (G5, Hamilton Medical, Bondauz, Switzerland) to maintain $\mathrm{SpO}_{2} 95-97 \%$ and $\mathrm{EtCO}_{2} 38-42 \mathrm{mmHg}$ at steady state according to the actual metabolic rate. Total intravenous anesthesia was maintained by continuous administration of propofol (6-12 mg/kg.h) and morphine (0.1-0.2 $\mathrm{mg} / \mathrm{kg} . \mathrm{h})$. The depth of anesthesia was regularly assessed by photoreaction, corneal reflex and Bispectral index monitoring (target values of 60-40). Intravenous infusion of normal saline was given to reach and maintain baseline central venous pressure between 4 and $6 \mathrm{mmHg}$. Anticoagulation was provided by unfractionated heparin bolus (100 IU/kg i.v.) followed by continuous intravenous drip (40-50 IU/kg/h) to maintain target activated clotting time (ACT) $180-250$ seconds (values were checked every hour with Hemochron Junior+, International Technidyne Corporation, Edison, NJ, USA). Sheaths and catheters were inserted into femoral and carotid/jugular vessels as needed. Invasive blood pressures from femoral and pulmonary artery, central venous pressure (TruWave, Edwards Lifesciences, USA), ECG, capnometry, pulse oximetry and rectal temperature were continuously monitored by bedside monitor (Life Scope TR, Nihon Kohden, Japan) and trends were recorded. Mixed venous oximetry $\left(\mathrm{SvO}_{2}\right)$, continuous cardiac output $(\mathrm{CCO})$ and pulmonary artery temperature were recorded by Swan-Ganz Combo V catheter (Vigilance monitor, Edwards Lifesciences, USA). Right ventricle was visualized by intracardiac echocardiographic (ICE) probe (Acuson X300, Siemens, USA). After acquiring an optimal projection of RV long axis with tricuspid valve and without the RV outflow tract, was the tip of ICE catheter fixed in right atrium and the actual projection through the experiment was regularly compared to the initial projection and adjusted as needed. RV end-diastolic area was evaluated by manual tracing of endocardium. Obstruction of pulmonary artery was provided by insertion of a large sizing balloon catheter under fluoroscopic guidance (size of the balloon $40 \mathrm{~mm}$ x $60 \mathrm{~mm}$, B. Braun Interventional Systems Inc., USA).

\section{Protocol}

The baseline data were averaged from three 
readings in 5 minute sequences at least 60 minutes after induction of general anesthesia and insertion of all the catheters. Afterwards, the obstruction procedures were performed by a stepwise inflation of pulmonary artery balloon catheter by diluted iodine contrast solution. Each step lasted 3 minutes and was managed to increase pulmonary artery mean pressure (PAMP) of $5 \mathrm{mmHg}$. The point of maximal hemodynamic compensatory steady state was defined as the last stable value of observed hemodynamic and ventilation parameters just before the next step leading to uncontrollable shock and cardiac arrest (fall of heart rate (HR) $<20 / \mathrm{min}$, mean arterial pressure (MAP) $<20 \mathrm{mmHg}, \mathrm{SpO}_{2}<60 \%$ ). After the hemodynamic collapse, the balloon was promptly deflated and the next obstruction procedure was commenced at least 10 minutes after stabilization of baseline hemodynamic values. In total, 10 obstruction procedures were performed in each of the experimental animals.

\section{Statistics}

Data are presented as median (interquartile range) of measured values or as median (interquartile range) of baseline percentage. Baseline data are established as $100 \%$. The median of baseline percentage is not percent expression of absolute median value, but it is a median of baseline percentage from each experiment. Repeated analysis of variance measures were used to assess the between-subject effect and no difference was noticed. Comparisons between baseline and maximal compensatory response data were performed using Mann-Whitney test, all $p$-values were two-tailed and $p<0.05$ was considered significant. Statistical analysis was performed using Statistica software version 8 (StatSoft, Tulsa, USA).

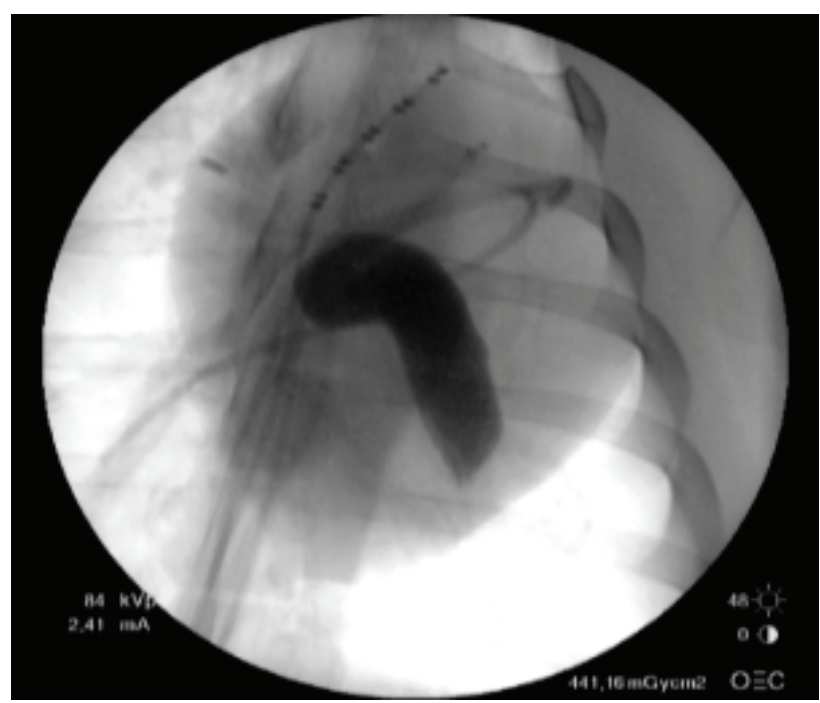

Fig. 1. Fluoroscopy of the balloon catheter wedged in pulmonary artery bifurcation.

\section{Results}

In the pre-study experiment we observed that: 1) total obstruction of isolated right or left pulmonary artery did not lead to hemodynamic collapse; 2) when the balloon catheter was wedged in pulmonary artery bifurcation, it obstructed both branches (Fig. 1) and hemodynamic collapse could be reached; 3 ) following the hemodynamic shock and immediate obstruction release, 10-minutes recovery period was sufficient to restore and stabilize hemodynamics and respiration parameters.

All observed hemodynamic parameters changed significantly at the point of maximal compensatory response (Fig. 2). The baseline RV end-diastolic area estimated by ICE was $11.6(10.1,14.1) \mathrm{cm}^{2}$ (Fig. 3). During the obstruction procedure RV end-diastolic area
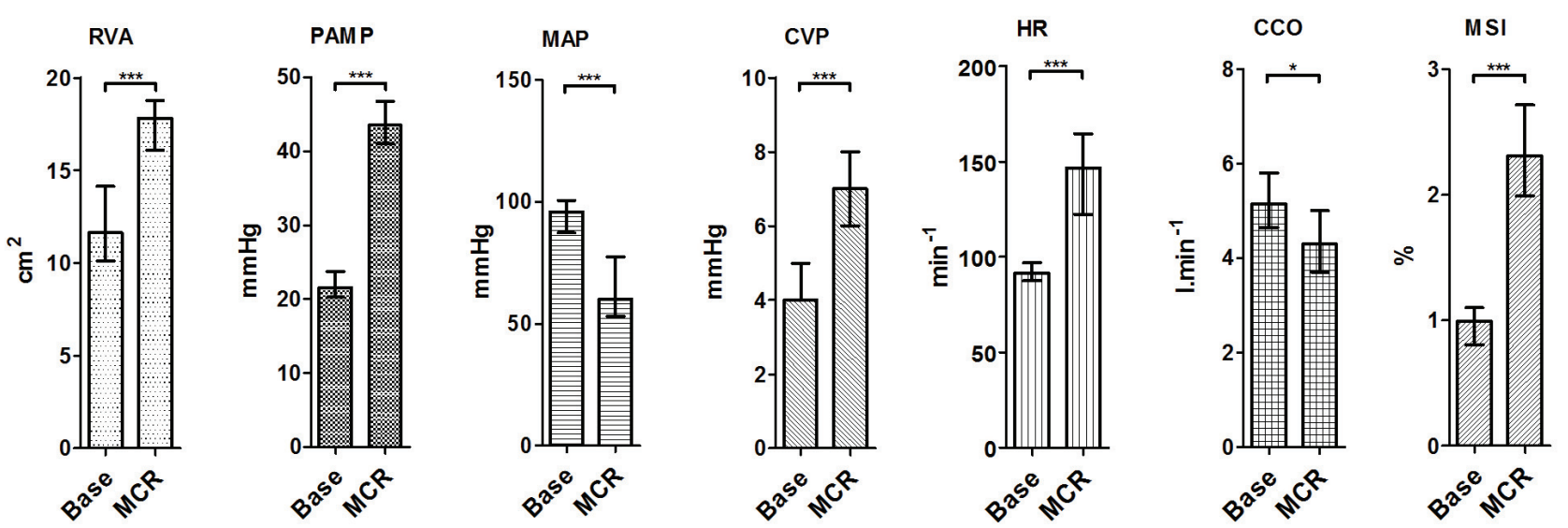

Fig. 2. Baseline (Base) and maximal compensatory response (MCR) median values of hemodynamic parameters; bars represent interquartile rage. All the changes were statistically significant $(* p<0.05, * * p<0.001, * * * p<0.0001)$. 


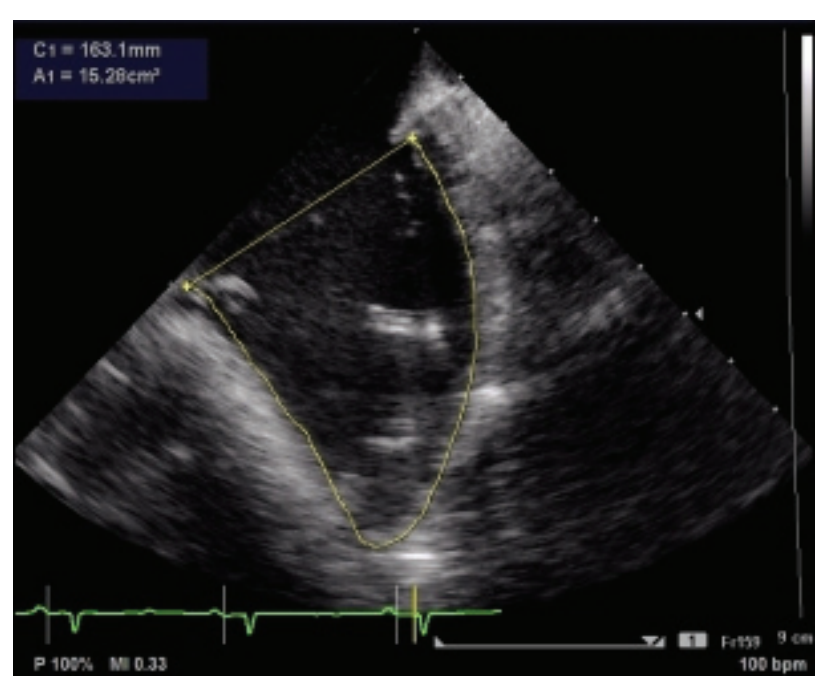

Fig. 3. Measuring of RV end-diastolic area by manual tracing of endocardium.

increased proportionally to PAMP $\left(0.4 \mathrm{~cm}^{2} / \mathrm{mmHg}\right.$ of PAMP; $\mathrm{p}<0.0001, \mathrm{R}^{2}=0.83$; Fig. 4). The maximum RV end-diastolic area reached $17.8(16.1,18.8) \mathrm{cm}^{2}$, $\mathrm{p}<0.0001 ; 150(135,159) \%$ of baseline coupled with severe systolic RV dysfunction and severe tricuspid valve regurgitation visible in color Doppler imaging mode. The tricuspid regurgitation also created positive " $\mathrm{x}$ " and " $\mathrm{v}$ " waves on CVP curve during the systole of RV (see example trace Fig. 5). PAMP increased from $22(20,24)$ $\mathrm{mmHg}$ to $44(41,47) \mathrm{mmHg}, \mathrm{p}<0.0001 ; 204(175$, 224) $\%$ of baseline. Accordingly, MAP decreased from $96(87,101) \mathrm{mmHg}$ to $60(53,78) \mathrm{mmHg}, \mathrm{p}<0.0001$; $61(60,74) \%$ of baseline, CVP increased from $4(4,5)$ mmHg to $7(6,8) \mathrm{mmHg}, \mathrm{p}<0.0001 ; 188(164,200) \%$ of baseline, and HR increased from $92(88,97)$ to $147(122$, $165)$ beats per minute, $\mathrm{p}<0.0001 ; 156(132,180) \%$ of baseline. CCO dropped from $5.2(4.7,5.8) 1 / \mathrm{min}$ to 4.3 $(3.7,5.0) \mathrm{l} / \mathrm{min}, \mathrm{p}=0.0023$ during the shock and seemed to correspond inadequately with large time delay compared to values measured by bolus injections. Estimated modified shock index (MSI), MAP divided by HR, increased from $0.99(0.81,1.10)$ to $2.31(1.99,2.72)$, $\mathrm{p}<0.0001$. After overcoming the maximal compensatory response point, uncontrollable hypoxia and retention of carbon dioxide appeared due to the loss of lung perfusion - HR dropped rapidly and, finally, electromechanic dissociation occurred. In all cases quick deflation of the balloon was followed by immediate restoration of spontaneous circulation (ROSC). In all cases the time of restitution of baseline steady state did not exceed 10 minutes.

\section{Dependence $\triangle$ RVA on $\triangle P A M P$}

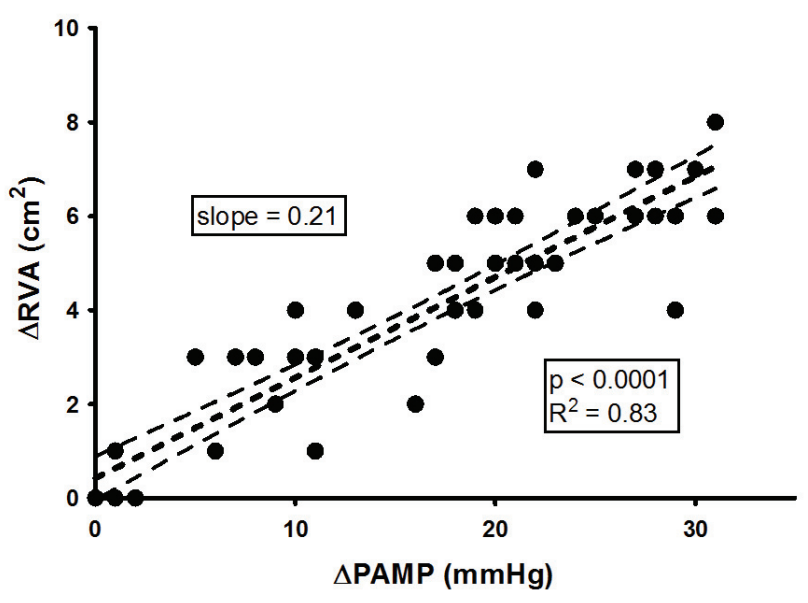

Fig. 4. Dependence of relative changes of RV end-diastolic area on PAMP. RV end-diastolic area increased proportionally at the rate $0.4 \mathrm{~cm}^{2} / \mathrm{mmHg}$ of PAMP $\left(p<0.0001, \mathrm{R}^{2}=0.83\right)$.

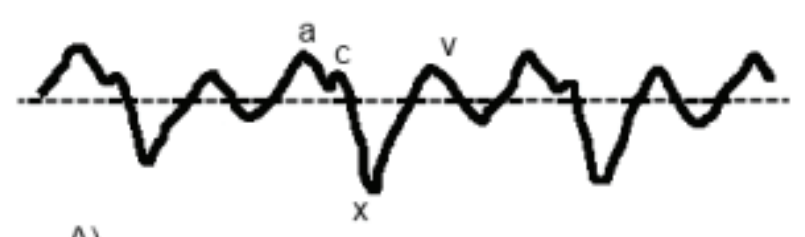

A)

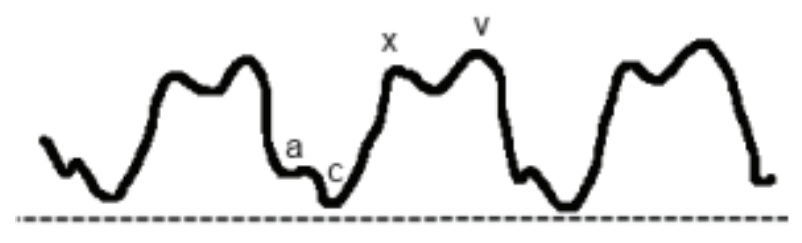

B)

Fig. 5. A) Baseline CVP curve. B) CVP curve during severe tricuspid valve regurgitation with positively inversed " $x$ " and " $v$ " waves.

\section{Discussion}

Standard risk stratification of patients with PE includes evaluation of hemodynamic status followed by laboratory and imaging methods. The high risk of sudden death is associated with the presence of shock, syncope or hypotension (systolic blood pressure below $90 \mathrm{mmHg}$, or drop of at least $40 \mathrm{mmHg}$ for more than 15 minutes). Normotensive patients with RV dysfunction and presence of markers of myocardial injury are also at higher risk (Kucher et al. 2006). Evaluation of RV dysfunction is in clinical practice routinely made by bedside transthoracic echocardiography, which remains the only imaging method in unstable patients. In addition, some authors 
mean that assessment of RV dysfunction is more accurate for risk stratification than only describing distribution and severity of vascular obstruction of clots in pulmonary circulation (ten Wolde et al. 2004). Some recent studies also highlight the role of multidetector computer tomography (MDCT) which can be also used to reveal right-to-left ventricular dimensional ratio as a sign of $\mathrm{RV}$ dysfunction (Hartmann et al. 2010, Henzler et al. 2011). On the other hand, lack of uniform echocardiographic criteria of RV dysfunction makes the exact stratification of the risk difficult.

In our study we measured RV end-diastolic area by ICE because of vertical orientation of the pig heart and using artificial ventilation, which both prevent keeping of precise and stable projection by transthoracic echocardiography through the experiment. The visualization of RV corresponds to inverse $\mathrm{RV}$ view in apical four-chamber projection in humans, which applies RV long axis view with tricuspid valve and without the $\mathrm{RV}$ outflow tract. On the contrary, ICE projection is limited by impossibility of visualization both right and left ventricles, so right-to-left ventricular dimensional ratio as a routinely used clinical parameter could not been measured. Based on our results, RV end-diastolic area seems to be very closely proportionally related to pulmonary artery pressure. The normal value of RV enddiastolic area in adult humans is between 11 and $28 \mathrm{~cm}^{2}$ (Lang et al. 2005), which corresponds to our median baseline value $11.6 \mathrm{~cm}^{2}$ (estimated body surface area (BSA) of the pigs was about $1 \mathrm{~m}^{2}$ ). According to maximal RV end-diastolic area at the peak of compensatory steady state (150\% of baseline) we can assume that the area of non-preconditioned RV can adapt up to $18 \mathrm{~cm}^{2} / \mathrm{m}^{2}$ in humans $\left(17-42 \mathrm{~cm}^{2}\right.$ according to BSA).

In this study was PAMP measured invasively, but in the clinical routine practice it can be estimated by echocardiography. The first choice is to measure the peak regurgitation pressure gradient via tricuspid valve (tracing of tricuspid valve regurgitation jet in continuous wave Doppler mode), and pulmonary artery systolic pressure (PASP) can be estimated by adding CVP. Then PAMP can be calculated using formula: PAMP = 0.61 PASP + 2 (Chemla et al. 2004). Similarly, the second possibility is to measure the peak regurgitation pressure gradient via pulmonary valve. Then PAMP is acquired as the sum of the peak regurgitation pressure gradient with CVP. As stated above, PAMP increased twice (up to $44 \mathrm{mmHg}$ ) which is concordant with previously published data (McIntyre et al. 1971, Toosi et al. 2008). The clinical observational study by Toosi (Toosi et al. 2008) identified the cut-off value PASP $>50 \mathrm{mmHg} \quad($ PAMP $>33 \mathrm{mmHg}) \quad$ significantly associated with increased in-hospital death, which corresponds to $75 \%$ of supposed maximal acute compensatory value. We suggest urgent echocardiographic assessment and regular monitoring of $\mathrm{RV}$ dysfunction is necessary in patients with moderate or high risk $P E$.

To avoid further hemodynamic destabilization, it is crucial to meet energetic and metabolic demands of myocardium, which includes restoration of pulmonary flow and preserving normoxemia. The contraindication or failure of thrombolysis in high risk patients along with exhaustion of compensatory mechanisms or next embolization strongly increases the risk of hemodynamic collapse. In such situation the patients could benefit from ECMO as a suitable bridge to overcome uncontrollable clinical status to acute surgical embolectomy or catheter based interventions. In addition, the previously reported case report documented improvement of hemodynamics of the high-risk patient contraindicated for thrombolysis or invasive procedures after using ECMO and administration of heparin anticoagulation therapy (Malekan et al. 2012).

Our data show that RV function seems to be very adaptable depending on pressure overload. In spite of contractility decline and dilatation of RV accompanied by severe tricuspid valve regurgitation leading to electromechanical dissociation, quick abolition of obstruction in pulmonary artery initiates quick restitution of normal size and function of RV. The design of the study was limited by relatively short duration of maximal compensatory steady states. To find out the long time stability of the system is necessary to design further experiments.

In conclusion, all the tested hemodynamic parameters except $\mathrm{CCO}$ are sensitive to assess the individual point of maximal acute hemodynamic compensatory steady state of the embolic obstructive shock (reaching $150 \%$ of RVA, $204 \%$ of PAMP, $61 \%$ of MAP and $156 \%$ of HR basal values). We suppose reaching $75 \%$ of predicted maximal value of pulmonary artery pressure (PASP $>50 \mathrm{mmHg} / \mathrm{PAMP}>33 \mathrm{mmHg}$ ) should be considered as critical in normal unpreconditioned heart. Proper hemodynamic monitoring including regular assessment of RV function is necessary to recognize the critical phase of obstructive shock and to 
early use of more aggressive therapeutic procedures to avoid cardiac arrest and to improve the chance of survival.

\section{Conflict of Interest}

There is no conflict of interest.

\section{Acknowledgements}

Authors thank to Alena Ehrlichova, Karel Kypta and Tereza Vavrikova for invaluable technical and lab assistance. Supported by PRVOUK-P38/LF1/10, SVV2012-264504 and GAUK 420911.

\section{References}

AUJESKY D, OBROSKY DS, STONE RA, AUBLE TE, PERRIER A, CORNUZ J, ROY PM, FINE MJ: Derivation and validation of a prognostic model for pulmonary embolism. Am J Respir Crit Care Med 172: 1041-1046, 2005.

BARTLETT RH, GATTINONI L: Current status of extracorporeal life support (ECMO) for cardiopulmonary failure. Minerva Anestesiol 76: 534-540, 2010.

CHEMLA D, CASTELAIN V, HUMBERT M, HEBERT JL, SIMONNEAU G, LECARPENTIER Y, HERVE P: New formula for predicting mean pulmonary artery pressure using systolic pulmonary artery pressure. Chest 126: 1313-1317, 2004.

DALLA-VOLTA S, PALLA A, SANTOLICANDRO A, GIUNTINI C, PENGO V, VISIOLI O, ZONZIN P, ZANUTTINI D, BARBARESI F, AGNELLI G, ET AL.: PAIMS 2: alteplase combined with heparin versus heparin in the treatment of acute pulmonary embolism. Plasminogen activator Italian multicenter study 2. J Am Coll Cardiol 20: 520-526, 1992.

DAVIES MJ, ARSIWALA SS, MOORE HM, KERR S, SOSNOWSKI AW, FIRMIN RK: Extracorporeal membrane oxygenation for the treatment of massive pulmonary embolism. Ann Thorac Surg 60: 1801-1803, 1995.

HARTMANN IJ, WITTENBERGR, SCHAEFER-PROKOP C: Imaging of acute pulmonary embolism using multidetector CT angiography: an update on imaging technique and interpretation. Eur J Radiol 74: 40-49, 2010.

HENZLER T, BARRAZA JM Jr, NANCE JW Jr, COSTELLO P, KRISSAK R, FINK C, SCHOEPF UJ: CT imaging of acute pulmonary embolism. J Cardiovasc Comput Tomogr 5: 3-11, 2011.

HSIEH PC, WANG SS, KO WJ, HAN YY, CHU SH: Successful resuscitation of acute massive pulmonary embolism with extracorporeal membrane oxygenation and open embolectomy. Ann Thorac Surg 72: 266-267, 2001.

KASPER W, KONSTANTINIDES S, GEIBEL A, OLSCHEWSKI M, HEINRICH F, GROSSER KD, RAUBER K, IVERSEN S, REDECKER M, KIENAST J: Management strategies and determinants of outcome in acute major pulmonary embolism: results of a multicenter registry. J Am Coll Cardiol 30: 1165-1171, 1997.

KEARON C, KAHN SR, AGNELLI G, GOLDHABER S, RASKOB GE, COMEROTA AJ; AMERICAN COLLEGE OF CHEST PHYSICIANS: Antithrombotic therapy for venous thromboembolic disease: American College of Chest Physicians Evidence-Based Clinical Practice Guidelines (8th Edition). Chest 133: 454S-545S, 2008.

KUCHER N, ROSSI E, DE ROSA M, GOLDHABER SZ: Massive pulmonary embolism. Circulation 113: 577-582, 2006.

LANG RM, BIERIG M, DEVEREUX RB, FLACHSKAMPF FA, FOSTER E, PELLIKKA PA, PICARD MH, ROMAN MJ, SEWARD J, SHANEWISE JS, SOLOMON SD, SPENCER KT, SUTTON MS, STEWART WJ: Recommendations for chamber quantification: a report from the American Society of Echocardiography's Guidelines and Standards Committee and the Chamber Quantification Writing Group, developed in conjunction with the European Association of Echocardiography, a branch of the European Society of Cardiology. J Am Soc Echocardiogr 18: 1440-1463, 2005.

LEACCHE M, UNIC D, GOLDHABER SZ, RAWN JD, ARANKI SF, COUPER GS, MIHALJEVIC T, RIZZO RJ, COHN LH, AKLOG L, BYRNE JG: Modern surgical treatment of massive pulmonary embolism: results in 47 consecutive patients after rapid diagnosis and aggressive surgical approach. J Thorac Cardiovasc Surg 129: 1018-1023, 2005. 
LEVINE M, HIRSH J, WEITZ J, CRUICKSHANK M, NEEMEH J, TURPIE AG, GENT M: A randomized trial of a single bolus dosage regimen of recombinant tissue plasminogen activator in patients with acute pulmonary embolism. Chest 98: 1473-1479, 1990.

MALEKAN R, SAUNDERS PC, YU CJ, BROWN KA, GASS AL, SPIELVOGEL D, LANSMAN SL: Peripheral extracorporeal membrane oxygenation: comprehensive therapy for high-risk massive pulmonary embolism. Ann Thorac Surg 94: 104-108, 2012.

MCINTYRE KM, SASAHARA AA: The hemodynamic response to pulmonary embolism in patients without prior cardiopulmonary disease. Am J Cardiol 28: 288-294, 1971.

MENEVEAU N, SERONDE MF, BLONDE MC, LEGALERY P, DIDIER-PETIT K, BRIAND F, CAULFIELD F, SCHIELE F, BERNARD Y, BASSAND JP: Management of unsuccessful thrombolysis in acute massive pulmonary embolism. Chest 129: 1043-1050, 2006.

MOLLOY WD, LEE KY, GIRLING L, SCHICK U, PREWITT RM: Treatment of shock in a canine model of pulmonary embolism. Am Rev Respir Dis 130: 870-874, 1984.

SUKHIJA R, ARONOW WS, LEE J, KAKAR P, MCCLUNG JA, LEVY JA, BELKIN RN: Association of right ventricular dysfunction with in-hospital mortality in patients with acute pulmonary embolism and reduction in mortality in patients with right ventricular dysfunction by pulmonary embolectomy. Am J Cardiol 95: 695-696, 2005.

TEN WOLDE M, SOHNE M, QUAK E, MAC GILLAVRY MR, BULLER HR: Prognostic value of echocardiographically assessed right ventricular dysfunction in patients with pulmonary embolism. Arch Intern Med 164: 1685-1689, 2004.

TOOSI MS, MERLINO JD, LEEPER KV: Prognostic value of the shock index along with transthoracic echocardiography in risk stratification of patients with acute pulmonary embolism. Am J Cardiol 101: 700-705, 2008.

TORBICKI A, PERRIER A, KONSTANTINIDES S, AGNELLI G, GALIE N, PRUSZCZYK P, BENGEL F, BRADY AJ, FERREIRA D, JANSSENS U, KLEPETKO W, MAYER E, REMY-JARDIN M, BASSAND JP; ESC COMMITTEE FOR PRACTICE GUIDELINES (CPG): Guidelines on the diagnosis and management of acute pulmonary embolism: the Task Force for the Diagnosis and Management of Acute Pulmonary Embolism of the European Society of Cardiology (ESC). Eur Heart J 29: 2276-2315, 2008.

WEINBERG L, KAY C, LISKASER F, JONES D, TAY S, JAFFE S, SEEVANAYAGAM S, DOOLAN L: Successful treatment of peripartum massive pulmonary embolism with extracorporeal membrane oxygenation and catheter-directed pulmonary thrombolytic therapy. Anaesth Intensive Care 39: 486-491, 2011.

WICKI J, PERRIER A, PERNEGER TV, BOUNAMEAUX H, JUNOD AF: Predicting adverse outcome in patients with acute pulmonary embolism: a risk score. Thromb Haemost 84: 548-552, 2000. 